\title{
Estimation of Stochastic Attribute-Value Grammars using an Informative Sample
}

\author{
Miles Osborne \\ osborne@let.rug.nl \\ Rijksuniversiteit Groningen, The Netherlands*
}

\begin{abstract}
We argue that some of the computational complexity associated with estimation of stochastic attributcvalue grammars can be reduced by training upon an informative subset of the full training set. Results using the parsed Wall Strect Journal corpus show that in some circumstances, it is possible to obtain better estimation results using an informative sample than when training upon all the available material. Further experimentation demonstrates that with unlexicalised models, a Gaussian prior can reduce overfitting. However, when models are lexicalised and contain overlapping features, overfitting does not seem to be a problem, and a Gaussian prior makes minimal difference to performance. Our approach is applicable for situations when there are an infeasibly large number of parses in the training set, or else for when recovery of these parses from a packed representation is itself computationally expensive.
\end{abstract}

\section{Introduction}

Abney showed that attribute-value grammars cannot be modelled adequately using statistical techniques which assume that statistical dependencies are accidental (Abney, 1997). Instead of using a model class that assumed independence, Abncy suggested using Random Fields Models (RFMs) for attribute-value grammars. RFMs deal with the graphical structure of a parse. Because they do not make independence assumptions about the stochastic generation process that might have produced some parse, they are able to model correctly dependencies that exist within parses.

When estimating standardly-formulated RFMs, it is necessary to sum over all parses licensed by the grammar. For many broad coverage natural language grammars, this might involve summing over an exponential number of parses. This would make the task computationally intractable. Abncy, following the lead of Lafferty $e t$ al, suggested a Monte

\footnotetext{
* Current address: osborne@cogsci.ed.ac.uk, University of Edinburgh, Jivision of Informatics, 2 Buccleuch l'lace, EH8 9LW, Scotland.
}

Carlo simulation as a way of reducing the computational burden associated with RFM estimation (Lafferty et al., 1997). However, Johnson et al considered the form of sampling used in this simulation (Metropolis-Hastings) intractable (Johnson et al., 1999). Instead, thcy proposed an alternative strategy that redefined the estimation task. It, was argued that this redefinition made estimation computationally simple cnough that a Monte Carlo simulation was unnecessary. Thcy prosented results obtained using a small unlexicalised model trained on a modest corpus.

Unfortunately, Johnson et al assumed it was possible to retrieve all parses licensed by a grammar when parsing a given training set. For us, this was not the case. In our experiments with a manually written broad coverage Definite Clause Grammar (DCG) (Briscoc and Carroll, 1996), we were only alsle to recover all parses for Wall Street Journal sentences that were at most 13 tokens long within acceptable time and space bounds on computation. When we used an incremental Minimum Description Length (MDL) based learner to extend the coverage of our manually written grammar (from roughly $60 \%$ to around $90 \%$ of the parsed Wall Strcet Journal), the situation became worse. Sentence ambiguity considerably increased. We were then only able to recover all parses for Wall Strect .Journal sentences that were at most 6 tokens long (Osborne, 1999).

We can however, and usually in polynomial time, recover up to 30 parses for sentences up to 30 tokens long when we use a probabilistic unpacking mechanism (Carroll and Briscoe, 1992). (Longer sentences than 30 tokens can be parsed, but the number of parses we can recover for them drops off rapidly). ${ }^{1}$ However, 30 is far less than the maximum number

\footnotetext{
${ }^{1}$ We made an attempt to determine the maximum number of parses our grammar might assign to sentences. On a $450 \mathrm{MHz}$ Ultra Sparc 80 with 2 Gb of real memory, with a limit of at most 1000 parses per sentence, and allowing no more than $100 \mathrm{CPU}$ seconds per sentence, we found that sentence ambiguity increased exponentially with respect to sentence length. Sentences with 30 tokens had an estimated average of 866 parses (standard deviation 290.4). Without the limit of 1000 parses per sentence, it seems likely that this average would increase.
} 
of parses per sentence onr grammar might assign to Wall Street. Journal sentences. Any training set we have access to will therefore be necessarily limited in size.

We thorefore noed an ostimation strategy that takes seriously the issue of extracting the best performance from a limitod size training set. $A$ limited size training set means one created by rotricving at most $n$ parses per sentence. Although we camnot recover all possiblo parses, we do have a choice as to which parses estimation should be based upon.

Our approach to the problem of making $R \mathrm{~F}$ M estimation feasible for our highly ambiguous I)CG is to seek out an informative sample and train upon that. We do not redefine the estimation task in a non-standard way, nor do wo use a Monte Carlo simsulation.

We call a sample informative if it both leads to the sclection of a model that does not underfit or overfit, and also is typical of future samples. Despite one's intuitions, an informative sample might be a proper subset of the full training set. This means that estimation using the informative sannple might, yield bettor results than estimation lusing all of tho training set,

The rest of this paper is ats follows. Jirstly we introduce RFM. Then we show how they may be estimated and how an informative sample might be identified. Next, we give details of the attributevalue grammar wo use, and show how we go about rodelling it. We then present, two sets of experiments. The first set is small scale, and are designed to show the existence of aje informative sample. The second set of experiments are larger in scale, and build upon the computational savings wo aro able to achieve using a probabilistic mpacking strategy. they show how large models (two orders of magnitude larger than those reported by Johnson of al) can be estimated using the parsed Wall Street Journal corpus. Overfitting is shown to take place. They also show how this overfiting can be (partially) reduced by using a Gaussian prior. Finally, we end with some comments on our work.

\section{Random Field Models}

Here we show how attribute-value grammars may be modelled using RFMs. Although our commentary is in terms of RFMs and grammars, it should be obvious that RFM technology can be applied to other estimation scenarios.

Let $G$ be an attribute-value grammar, $D$ the set of sentences within the string-set defined by $L(G)$ and $\Omega$ the union of the set of parses assigned to cach sentence in $D$ by the grammar $G$. A Random Fidd Modd, $M$, consist of two components: a set of features, $F$ and a set of weights, $\Lambda$.

Features are the basic building blocks of RTMs.
They cnable the system designor to specify the key aspects of what it talkes to differentiate one parse from another parse. Fach feature is a function from a parse to an integer. Here, the integer value associated with a feature is interpreted as the number of times a feature 'matches' (is 'active') with a parse. Note foatures should not be confused with features as found in foature-value bundles (these will bo called attributes instcad). Foatures are msually manually selected by the system designer.

The other component of a RFM, $\Lambda$, is a set of woights. Informally, woights tell us how features are to be used when modelling parses. For example, an active featine with a largo woight might indicate that some parse had a high probability. Fach weight $\lambda_{i}$ is associated with a feature $f_{i}$. Woights are real-valued numbers and are automatically determined by an estimation process (for example using Improved Iterative Scaling (Lafferty et al., 1997)). One of the nice properties of RIMs is that the likelihood function of a JRM is strictly concave. This moans that there are no local minima, and so wo can be be sure that scaling will result in estimation of a RFM that, is globally optimal.

The (unnormalised) total weight of a parse $x$, $\psi(x)$, is a function of the $k$ features that are 'active' on a parse:

$$
\psi(x)=\exp \left(\sum_{i=1}^{k} \lambda_{i} f_{i}(x)\right)
$$

The probability of a parse, $P(x \mid M)$, is simply the result of normalising the total weight associated with that parse:

$$
\begin{array}{r}
P(x \mid M)=\frac{1}{Z} y /(x) \\
Z=\sum_{y \in \Omega} \psi(y)
\end{array}
$$

The interpretation of this probability depends upor the application of the RFM. Here, we use parse probabilitics to reflect preforences for parses.

When using RFMs for parse selection, we simply select the parse that maximises $\psi(x)$. In these circumstances, there is no need to normalise (compute $Z$ ). Also, when computing $\psi(x)$ for competing parses, there is no built-in bias towards shorter (or longer) derivations, and so no need to normalise with respect to derivation longth. ${ }^{2}$

\footnotetext{
The reason there is no necl to normalise with respect to derivation length is that features can have positive or nega tive weights. The weight of a parse will therefore not always monotonically increase with respect to the number of active foatures.
} 


\section{$3 \quad$ RFM Estimation and Selection of the Informative Sample}

We now sketch how RFMs may be estimated and then outline how we seek out an informative sample.

We use Improved Iterative Scaling (IIS) to estimate RFMs. In outline, the IIS algorithm is as follows:

1. Start, with a reference distribution $R$, a set of features $F$ and a set of weights $\Lambda$. Let $M$ be the RFM defined using $F$ and $\Lambda$.

2. Initialise all weights to zero. This makes the initial model uniform.

3. Compute the expectation of each feature w.r.t R.

4. For each feature $f_{i}$

(a) Find a woight $\check{\lambda}_{i}$ that equates the expectation of $f_{i}$ w.r.t $R$ and the expectation of $f_{i}$ w.r.t $M$.

(b) Replace the old value of $\lambda_{i}$ with $\check{\lambda_{i}}$.

5. If the model has converged to $R$, output $M$.

6. Otherwise, go to step 4

The key step here is $4 a$, computing the expectations -of features w.r.t the RFM. This involves calculating the probability of a parse, which, as we saw from equation 2, requires a summation over all parses in $\Omega$.

Wo scek out an informative sample $\Omega_{i}\left(\Omega_{t} \subseteq \Omega\right)$ as follows:

1. Pick out from $\Omega$ a sample of size $n$.

2. Estimate a model using that sample and evaluate it.

3. If the model just estimated shows signs of overfitting (with respect to an unseen held-out data set), halt and output the model.

4. Otherwise, increase $n$ and go back to step 1 .

Our approach is motivated by the following (partially related) observations:

- Because we use a non-parametric model class and select an instance of it in terms of some sample (section 5 gives details), a stochastic complexity argument tells us that an overly simple model (resulting from a small sample) is likely to underfit. Likewise, an overly complex model (resulting from a large sample) is likely to overfit. An informative sample will therefore relate to a model that does not under or overfit.

- On average, an informative sample will be 'typical' of future samples. For many real-life situations, this set is likely to be small relative to the size of the full training set.
We incorporate the first observation through our search mechanism. Because we start with small samples and gradually increase their size, we remain within the domain of efficiently recoverable samples.

The second observation is (largely) incorporated in the way we pick samples. The experimental section of this paper goes into the relevant details.

Note our approach is heuristic: we cannot afford to evaluate all $2^{|\Omega|}$ possible training sets. The actual size of the informative sample $\Omega_{t}$ will depend both the upon the model class used and the maximum sentence length we can deal with. We would expect richer, lexicalised models to exhibit overfitting with smaller samples than would be the case with unlexicalised models. Wo would expect the size of an informative sample to increase as the maximum sentence length increased.

There are similarities between our approach and with estimation using MDI (Rissancn, 1989). Howcver, our implementation does not; explicitly attempt to minimise code lengths. Also, there are similarities with importance sampling approaches to RFM estimation (such as (Chen and Rosenfeld, 1999a)). However, such attempts do not minimise under or overfitting.

\section{The Grammar}

The grammar we model with Random Fields, (called the Tag Sequence Grammar (Briscoe and Carroll, 1996), or 'TSG for short) was developed with regard to coverage, and when compiled consists of 455 Definite Clause Grammar (DCG) rules. It cloes not parse sequences of words directly, but instead assigns derivations to sequences of part-of-specch tag's (using the CLAWS2 tagset. The grammar is rolatively strallow, (for example, it does not fully analyse unbounded dependencies) but it does make an attompt to doal with common constructions, such as dates or names, commonly found in corpora, but of little theoretical interest. Furthermore, it integrates into the syntax a text grammar, grouping utiterances into units that reduce the overall ambiguity.

\section{Modelling the Grammar}

Modelling the TSG with respect to the parsed Wall Strect Journal consists of two steps: creation of a feature set and definition of the reference distribution.

Our feature set is created by parsing sentences in the training set $\left(\Omega_{T}\right)$, and using each parse to instantiate templates. Each template defines a family of features. At present, the templates we use are somewhat ad-hoc. However, they are motivated by the observations that linguistically-stipulated units (DCG rules) are informative, and that many DCG applications in preferred parses can be predicted using lexical information. 


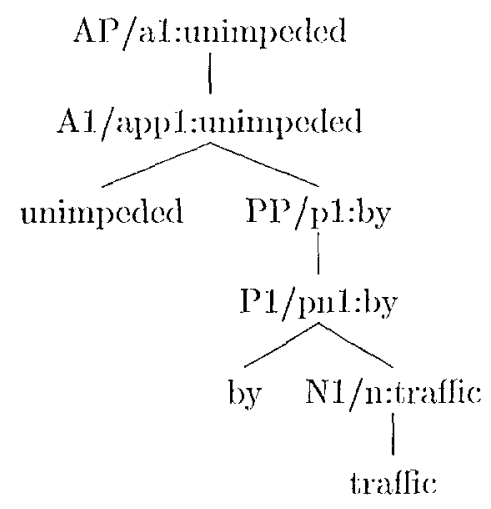

Figure 1: 'TSG Parse Fragment

The first template creates features that connt the number of times a DCG instantiationis present within a parse." For example, suppose wo parsed the Wall Street Journal AP:

1 unimpeded by traffic:

A parse treo generated by TSG might be as shown in figure 1. Here, to save on space, we have labelled cach interior node in the parse tree with TSG rule names, and not, attribute-value bundles. Furthermore, we have annotated each node with the hoad word of the phrase in question. Within om grammar, hoads are (usually) explicitly marked. This means wo do not have to make any guesses when identifying the head of a local trec. With head information, we are able to lexicalise models. Wo have suppressed tagging information.

For example, a feature defined using this tomplate might count the number of times the we saw:

$$
\mathrm{Al}_{\mathrm{Al} / \mathrm{app} 1}
$$

in a parse. Such features record some of the context of the rule application, in that rule applications that differ in terms of how attributes are bound will be modelled by difforent features.

Oul: second template creates features that are partially lexicalised. For oach local trec (of depth one) that has a PP daughter, we croate a feature that counts the number of times that local trec, clecorated with the head-word of the I'P', was seen in a parse. An example of such a lexicalised feature would bo:

$$
\mathrm{PP}_{\mathrm{Pl} 1 \mathrm{p} \mathrm{by}}^{\mathrm{Al} \text { appl }}
$$

\footnotetext{
${ }^{3}$ Note, all our features suppress any teminals that appea in a local tree. Iexical information is included when we decide to lexicalise features.
}

These features are designed to model PP attachments that can be resolved using the head of the Pl?

The third and final template creates features that are again partially lexicalised. This time, we create local trees of depth one that are decorated with the head word. For example, here is one such feature:

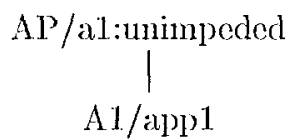

Note the second and third templates result in features that overlap with features resulting from applications of the first template.

We create the reference distribution $R$ (an association of probabilitios with TSG parses of sentences, such that the probabilitics reflect parse preferences) using the following process:

1. Extract some sample $\Omega_{T}$ (using the approach montioned in section 3).

2. For each sentence in the sample, for each parse of that sentence, compute the 'distance' between the TSG parse and the WS.J reference parse. In our approach, distatnce is calculated in terms of a weighted sum of crossing rates, recall and precision. Minimising it maximises our definition of parse plausibility. However, there is nothing inherently crucial about this decision. Any other objective function (that can be represented as an exponential distribution) could be nsed instoud.

3. Nomalise the distances, such that for some sentence, the sum of the distances of all recovered TSG parses for that sentence is a constant, across all sentences. Normalising in this mantner cnsures that each sentence is equiprobable (remember that; RFM probabilities are in terms of parse preforences, and not probibility of occurrence in some corpus).

4. Map the nomalised distancos into probabilities. If $d(p)$ is the normalised distance of TSG parse $p$, then associate with parse $p$ the referenco probability given by the maximum likelihood estimator:

$$
\frac{d(p)}{\sum_{x \in \Omega,} d(x)}
$$

Our approach therefore gives partial credit (a nonzero reference probability) to all parses in $\Omega_{t} . R$ is therefore not as discontinuous as the equivalent distribution used by Johnson et al. We therefore do not need to use simulated annealing or other numerically intensive techniques to estimate models.

\footnotetext{
${ }^{4}$ Our distance metric is the same one used by lJektoen (Hektoen, 1997)
} 


\section{$6 \quad$ Experiments}

Here we prosent two sets of experiments. The first set demonstrate the existence of an informative sample. It also shows some of the characteristics of three sampling stratcgies. The sccond set of experiments is larger in scale, and show RFMs (both lexicalised and unlexicalised) estimated using sentences up to 30 tokens long. Also, the effects of a Gaussian prior are demonstrated as a way of (partially) dealing with overfitting.

\subsection{Testing the Various Sampling Strategies}

In order to see how various sizes of sample related to estimation accuracy and whether we could achieve similar levels of performance without recovering all possible parses, we ran the following experimonts.

We used a model consisting of features that were defined using all three templates. Wo also threw away all features that occurred less than two times in the training set. We randomly split the Wall Street Journal into disjoint training, held-out and testing sets. All sentences in the training and held-out sets were at most, 14 tokens long. Sentences in the testing set were at most 30 tokens long. There were 6626 sentences in the training set, 98 sentences in the hold-out set and 441 sentences in the testing set. Sentences in the held-out set had on average 12.6 parses, whilst sentences in the testing-set had on average 60.6 parses per sentence.

The held-out set was used to decide which model performed best. Actual performance of the models should be judged with respect to the testing set.

Evaluation was in terms of exact match: for each sentence in the test set, we awarded ourselves a point, if the RFM ranked highest the same parse that was ranked highest using the reference probabilities. When evaluating with respect to the hold-out set, we recovered all parses for sontences in the held-out set. When evaluating with respect to the testing-set, we recovered at most 100 parses per sentence.

For cach run, we ran IIS for the same number of iterations (20). In each case, we evaluated the RFM after each other iteration and recorded the best classification performance. This step was designed to avoid overfitting distorting our results.

Figure 2 shows the results we obtained with possible ways of picking 'typical' samples. The first column shows the maximum number of parses per sentences that we retrieved in each sample.

The second column shows the size of the sample (in parses).

The other columns give classification accuracy results (a percentage) with respect to the testing set. In parentheses, we give performance with respect to the held-out set.

The column marked Rand shows the performance

\begin{tabular}{ll|lll} 
Max parses & Size & Rand & SCFG & Ref \\
\hline 1 & 6626 & $25.2(51.7)$ & $23.3(50.0)$ & $23.4(50.0)$ \\
2 & 12331 & $37.9(63.0)$ & $40.4(60.3)$ & $40.4(60.0)$ \\
3 & 17026 & $43.2(65.5)$ & $43.7(63.8)$ & $43.7(63.8)$ \\
5 & 24878 & $43.7(70.2)$ & $45.8(69.5)$ & $45.8(69.5)$ \\
$\mathbf{1 0}$ & $\mathbf{3 9 5 8 1}$ & $\mathbf{4 7 . 4 ( 7 2 . 0 )}$ & $\mathbf{4 7 . 0 ( 7 0 . 0 )}$ & $\mathbf{4 6 . 9 ( 7 0 . 0 )}$ \\
100 & 119694 & $45.0(68.7)$ & $45.0(68.0)$ & $45.0(68.0)$ \\
1000 & 246686 & $44.4(67.4)$ & $43.0(67.0)$ & $43.0(67.0)$ \\
$\infty$ & 267400 & $43.0(66.0)$ & $43.0(66.0)$ & $43.0(66.0)$
\end{tabular}

Figure 2: Results with various sampling strategies

of runs that used a sample that contained parses which were randomly and uniformly sclected out of the set of all possible parses. The classification accuracy results for this sampler are averaged over 10 runs.

The column marked $S C F G$ shows the results obtainod when using a sample that contained parses that were retrieved using the probabilistic unpacking strategy. This did not involve retrieving all possible parses for each sentence in the training set. Since there is no random component, the results are from a single run. Here, parses were ranked using a stochastic context free backbone approximation of TSG. Parameters were estimated using simple counting.

Finally, the columm marked Ref shows the results obtained when using a sample that contained the overall $n$-best parses per scntence, as defined in terms of the reference distribution.

As a bascline, a model containing randomly assigned woights produced a classification accuracy of $45 \%$ on the held-out sentences. These results were averaged over 10 runs.

As can be seen, increasing the sample size produces better results (for each sampling strategy). Around a sample size of $40 k$ parses, overfitting starts to manifest, and performance bottoms-out. One of these is therefore our informative sample. Note that the best sample ( $40 k$ parses) is less than $20 \%$ of the total possible training set.

The difference between the various samplers is marginal, with a slight preforence for Rand. However the fact that $S C F G$ sampling scems to do almost as well as Rand sampling, and furthermore does not require unpacking all parses, makes it the sampling strategy of choice.

$S C F G$ sampling is biased in the sense that the sample produced using it will tend to concentrate around those parses that are all close to the best parses. Rand sampling is unbiased, and, apart from the practical problems of having to recover all parses, might in some circumstances be better than $S C F G$ sampling. At the time of writing this paper, it was unclear whether we could combine $S C F G$ with Rand sampling -sample parses from the full distribu- 
tion withont unpacking all parses. Wo suspect that for probabilistic umpacking to be efficient, it must rely upon some non-uniform distribution. Unpacking randomly and uniformly would probably result in a large loss in computational efficiency.

\subsection{Larger Scale Evaluation}

Here we show results using a larger sample and testing set. We also show the effects of lexicalisation, overfitting, and overfitting avoidance using a Gaussian prior. Strictly speaking this section could have been omitted from the paper. However, if one views cstimation using an informative sample as overfitting avoidance, then estimation using a Gaussian prior can be scen as another, complemontary take on the problem.

The experimental setup was as follows. We randomly split the Wall Strect Journal corpus into a training set and a testing set. Both sets contained sentences that were at most 30 tokens long. When creating the set of parses used to estimate RFMs, wo used the SCFG approach, and retained the top 25 parses per sentence. Within the training set (arising from 16,200 sentences), there were 405,020 parses. The tosting set consisted of 466 sentences, with an average of 60.6 parses per sentence.

When evaluating; we rotieved at most 100 parses pere sontence in the testing set and scored them using our reference distribution. As before, we awarded ourselves a point if the most probable testing parse (in terms of the RMF) coincided with the most probable parse (in terms of the reforence distribution). In all cases, we ran JIS for 100 iterations.

For the first experinent, we used just the first template (foatures that related to DCG instantiations) to croato model 1; the second experiment used the first and second templates (additional featiures relating to P'P attachment) to (roate model 2. The final experiment used all thee templates (additional features that were head-lexicalised) to create model 3.

The three models contained 39,230, 65,568 and 273,127 features respectively,

As a baseline, a model containing randomly assigned weights achieved a $22 \%$ classification accuracy. These results were averaged over 10 runs. Fig1ure 3 shows the classification accuracy using modols 1,2 and 3 .

As can be seen, the larger scale experimental results were better than those achicved using the smaller samples (mentioned in section 6.1). The reason for this was because we used longer sentences. The informative sample derivable from such a training sot was likely to be larger (more representiative of

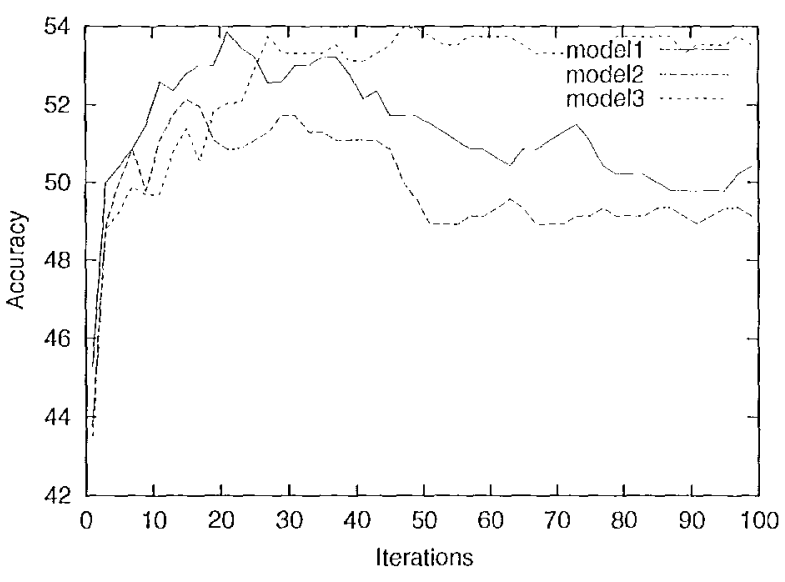

Figure 3: Classification Accuracy for Three Models Estimated using Basic IIS

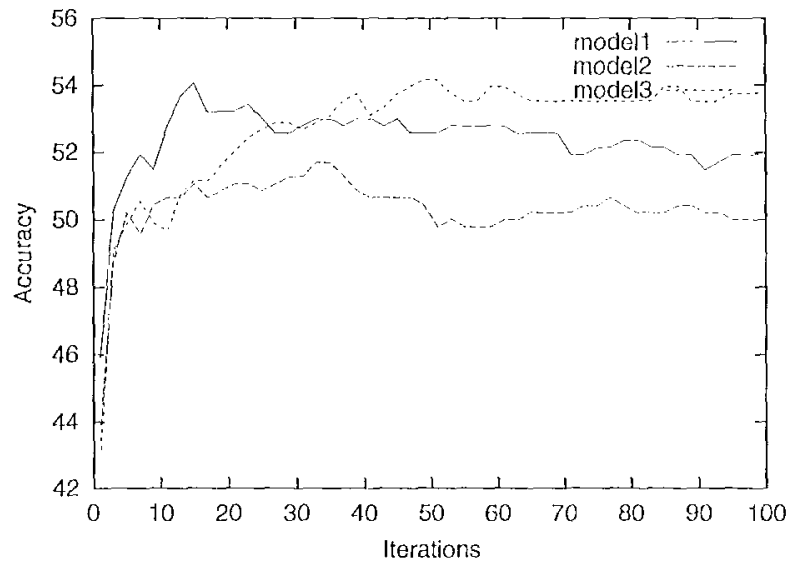

Figure 4: Classification Accuracy for 'Threc Models Estimated using a Ganssian Prior and IIS

the population) than the informative sample derivabled from a training set using shorter, less syntactically complex sentences. With the unlexicalised model, we see cloar signs of overfiting. Model 2 overfits even more so. For reasons that are unclear, we see that the larger model 3 does not appear to exhibit overfitting.

We next used the Gaussian Prior method of Chen and Rosenfeld to reduce overfitting (Chen and Rosenfeld, 1999b). This involved integrating a Gaussian prior (with a zero mean) into IIS and searching for the model that maximised the product of the likelihood and prior probabilities. For the experiments reported here, we used a single variance over the entire model (better results might be achicvable if multiple variances were used, perhaps with one variance per template type). 'Ihe actual value of the variance was found by trial-and-crror. However, optimisation using a hold-out set is easy to achicere. 
We repeated the large-scale experiment, but this time using a Gaussian prior. Figure 4 shows the classification accuracy of the models when using a Gaussian Prior.

When we used a Gaussian prior, we found that all models showed signs of improvement (allbeit with varying degrees): performance either increased, or else did not decrease with respect to the number of iterations. Still, model 2 continued to underperform. Model 3 seemed most resistent to the prior. It therefore appears that a Gaussian prior is most uscful for unlexicalised models, and that for models built from complex, overlapping features, other forms of smoothing must be used instead.

\section{Comments}

Wo argued that RFM estimation for broad-coverage attribute-valued grammars could be made computationally tractable by training upon an informative sample. Our small-scale experiments suggested that using those parses that could be efficiently unpacked (SCFG sampling) was almost as offective as sampling from all possible parses (Rand sampling). Also, we saw that models should not be both built and also estimated using all possible parses. Better results can bo obtained whon modols are built and trained using an informative sample.

Given the relationship between sample size and model complexity, we see that, when there is a danger of overfitting, one should build models on the basis of an informative set. However, this leaves open the possibility of training such a model upon a superset of the informative set. Although we have not tested this scenario, we bolice that this would lead to better results than those achieved hore.

The larger scale cxperiments showed that RFMs can be estimated using relatively long sentences. They also showed that a sinple Gaussian prior could reduce the effects of overfitting. However, they also showed that excessive overfitting probably required an altcrnative smoothing approach.

The smaller and larger experiments can be both vicwed as (complementary) ways of dealing with overfitting. We conjecture that of the two approaches, the informative sample approach is preferable as it deals with overfitting directly: overfitting results from fitting to complex a model with too litthe data.

Our ongoing research will concentrate upon stronger ways of dealing with overfitting in lcxicalised RFMs. One line we are pursuing is to combine a compression-based prior with an exponential model. This blends MDL with Maximum Entropy.

We are also looking at allemative template sets. For example, we would probably bencfit from using templates that capture more of the syntactic context of a rule instantiation.

\section{Acknowledgments}

We would like to thank Rob Malouf, Donnla Nic Gearailt and the anonymous reviewers for comments. This work was supported by the TMR Project Learning Computational Grammars.

\section{References}

Steven P. Abney. 1997. Stochastic AttributeValue Grammars. Computational Linguistics, 23(4):597-618, December.

Miles Osborne 1999. DCG Induction using MDL and Parsed Corpora. In James Cussens, editor, Learning Language in Logic, pages 63-71, Bled, Slovenia, Jume.

Ted Briscoe and John Carroll. 1996. Automatic Extraction of Subcatcgorization from Corporal. In Proceedings of the $5^{\text {th }}$ Conference on Applied NLP, pages 356-363, Washington, DC.

John Carroll and Ted Briscoe. 1992. Probabilistic Normalisation and Unpacking of Packed Parse Forests for Unification-based Grammars. In Procecdings of the AAAI Fall Symposium on Probabilistic Approaches to Natural Language, pages 33-38, Cambridge, MA.

Stanley Chen and Ronald Rosenfeld. 1999a. Eflicient Sampling and Fenture Selection in Whole Sentence Maximum Entropy Language Models. In ICASSI'99.

Stanley F. Chen and Ronald Rosenfold. 1999b. A Gaussian Prior for Smoothing Maximum Entropy Models. Technical Report CMU-CS-99-108, Carnegie Mellon University.

Firik Hektoen. 1997. Probabilistic Parse Selection Based on Semantic: Cooccurrences. In Procedings of the 5th International Worskhop on Parsing Technologies, Cambridge, Massachusetts, pages 113-122.

Mark Johnson, Stuart Geman, Stephen Cannon, Zhiyi Chi, and Stephan Riezler. 1999. Estimations for Stochastic "Unification-based" Grammars. In $37^{\text {th }}$ Annusl Mecting of the ACL.

J. Lafferty, S. Della Pietra, and V. Della Pictra. 1997. Inducing Features of Random Ficlds. IEEE Transactions on Pattern Analysis and Machine Intelligence, 19(4):380--393, April.

Jorma Rissancn. 1989. Stochastic Complexity in Statistical Inquiry. Scrics in Computer Scionce Volume 15. World Scientific. 